\title{
Tracheoesophageal Fistula Closed by Chemoradiotherapy in Lung Cancer
}

\author{
Takayuki Honda $^{a}$ Yoshihito Tsuzaki ${ }^{a}$ Keiko Mitaka ${ }^{a}$ \\ Kazuhiro Fukasawa ${ }^{a}$ Yoshihiro Miyashita $^{a}$ Kan Marino $^{b}$ \\ Akitoshi Saito $^{\text {b }}$ Toshio Oyamac Naohiko Inase $^{\mathrm{d}}$ \\ Departments of a Respiratory Medicine, ${ }^{\mathrm{b}}$ Radiology, and 'Pathology, Yamanashi \\ Prefectural Central Hospital, Kofu, and dDepartment of Integrated Pulmonology, \\ Tokyo Medical and Dental University, Tokyo, Japan
}

\section{Key Words}

Chemoradiotherapy · Lung carcinoma · Tracheoesophageal fistula

\begin{abstract}
A 45-year-old man complaining of cough, dyspnea, and difficulty in swallowing was referred to our hospital. Chest CT scan showed a mediastinal mass compressing the trachea. He was diagnosed with poorly differentiated lung carcinoma by percutaneous needle biopsy. Bronchoscopy and upper gastrointestinal endoscopy revealed a tracheoesophageal fistula (TEF). Long-lasting febrile neutropenia made it impossible to continue chemotherapy, but a course of radiotherapy (total $61 \mathrm{~Gy}$ ) was completed. The next endoscopy revealed closure of the TEF. Chemoradiotherapy (CRT) has been reported to close TEF in esophageal cancer, but the risk of a CRT-induced worsening of the fistula has dissuaded physicians from using CRT to treat TEF in lung cancer patients. CRT may serve as a palliative treatment for TEF in lung cancer as well as esophageal cancer.
\end{abstract}

\section{Introduction}

Malignant tracheoesophageal fistula (TEF) is a serious complication of thoracic malignant diseases and is more common in esophageal cancer than in lung cancer [1]. TEF sometimes results in aspiration pneumonia, sepsis, and malnutrition associated with trouble in eating. Without proper and prompt treatment, life expectancy is estimated at about 6 weeks [1]. Stent intubation into the trachea and/or esophagus is a reliable treatment leading to improved quality of life [2]. Surgical bypass with gastrostomy is another option, though it might be too invasive in some patients with advanced cancer. Chemoradiotherapy (CRT) is a controversial option, as it has the potential to induce or 
worsen TEF as a side effect, especially in lung cancer. In esophageal cancer, meanwhile, some reports have described closure of TEFs after CRT [3,4].

Our group experienced a rare case of TEF that closed after CRT in a patient with poorly differentiated lung carcinoma. CRT seems to have good potential as a palliative treatment for TEF in lung cancer, as well.

\section{Case Report}

A 45-year-old man with complaints of cough, dyspnea, weight loss, and difficulty in swallowing was referred to our hospital. The symptoms had emerged 3 months earlier and he had been completely unable to eat for several days before his admission. On physical examination, a solid mass at the right neck was palpable, the right palpebral fissure was narrowed, and inspiratory stridor was auscultated. An enhanced chest CT scan revealed a small mass in the superior sulcus invading into the mediastinum (fig. 1). The sagittal view revealed a cavity within the mass and compression of the stenotic trachea by the mass, but there was no apparent communication between the esophagus and trachea. Laboratory data indicated mild normocytic anemia and elevated C-reactive protein. Among tumor markers, an elevation of cytokeratin 19 fragment and neuron-specific enolase (table 1) was noted. The patient was diagnosed with poorly differentiated lung carcinoma by percutaneous needle biopsy (fig. 2). The patient's respiratory condition was too fragile to permit endoscopic examination at this point.

Radiation therapy was commenced immediately, before confirmation of the pathological diagnosis, for fear of sudden asphyxiation by the occupying mass. Chemotherapy with cisplatin and docetaxel was added, but was discontinued when the patient developed febrile neutropenia requiring granulocytestimulating factor and antibiotics for several days. When his respiratory condition improved enough to permit bronchoscopy and upper gastrointestinal endoscopy, these modalities revealed a hole with ulcerative edges in the trachea and upper esophagus (fig. 3 ). He was diagnosed with malignant TEF on that basis. We surmised that palliative treatment would prevent complications of TEF, such as aspiration pneumonia. In fact, the patient had already developed aspiration pneumonia during his hospital stay. As the fistula was located in the upper esophagus $(20 \mathrm{~cm}$ below the incisive papilla), we anticipated that intubation of an expandable metallic stent to the esophagus would be deeply uncomfortable and intolerable. With airway stenting, an accidental insertion of the stent into the cavity inside the tumor was a concern. As the initial effect of radiation had improved the compression of the tumor to the trachea, the therapy was continued up to a total dose of $61 \mathrm{~Gy}$. About 2 weeks after the radiation therapy was completed, the patient's severe cough subsided for several days. Encouraged by the improvement, he attempted to eat sweets and managed to do so without coughing. A bronchoscopy a few days later revealed only one slit on the trachea, and upper gastrointestinal endoscopy showed a covering of normal epithelial tissue over the fistula (fig. 3). In the ensuing weeks he fully regained the ability to eat orally, his general condition improved remarkably, and he left our hospital.

\section{Discussion}

TEF is generally categorized as congenital or acquired. Traumas and malignancy, conditions that may be associated with side effects of CRT, are common causes of acquired TEF. As treatment, curative resection followed by repair of the trachea and/or esophagus is often chosen in congenital or non-malignant TEF [5]. Surgery is usually considered inappropriate in malignant TEF, as the patients tend to be in a bad condition and near the end of their lives. Esophageal bypass procedures for these patients have high morbidity and mortality. Palliative therapy should be the primary choice in terms of quality of life, and the therapeutic goals should be to restore the patency of the trachea by reducing the extrinsic compression and to enable these patients to take nutrition and fluid [2]. 
Intubation of a stent to the tracheobronchial tree and/or esophagus is somewhat safer than the other procedures and good for symptomatic relief. Some papers report high success rates in double stenting [6]. When it is difficult to place a double stent at the same time, a tracheal stent has priority because an esophageal stent alone may compress the trachea and elicit respiratory distress [2]. We abandoned tracheal stenting in the current case for fear of a sudden impaction of the stent into the cavity. To avoid the risk of tracheal compression by the rapidly growing mediastinal tumor, we decided to administer CRT therapy in spite of the danger that the TEF would worsen.

With recent progress in the treatment of advanced and/or metastatic non-small-cell lung cancer, molecular targeted agents such as gefitinib, erlotinib, and bevacizumab play significant roles in improving patient survival. But the standard therapy for locally advanced tumors not suited for surgery is radiotherapy combined with chemotherapy based on a platinum-containing drug. It remains controversial whether such agents should be added to radiotherapy in locally advanced cases [7]. Chemotherapy combined with the angiogenesis inhibitor bevacizumab, a monoclonal antibody to human vascular endothelial growth factor (VEGF), improves overall survival in colorectal cancer and nonsmall-cell lung cancer $[8,9]$. Although bevacizumab is recognized to be relatively safe, it is associated with the risk of hypertension, proteinuria, delayed wound healing, bowel perforation, and so on. A recent report suggests that bevacizumab added to CRT is associated with a higher incidence of TEF formation in both small-cell and non-small-cell lung carcinomas [10].

Most of the published papers on TEF usually address the condition in patients with esophageal cancer. Manabu et al. [11] reported that CRT brought about fistula closure in 17 of $24(70.8 \%)$ patients with esophageal cancer and led to restored oral alimentation in 16 of those $17(94.1 \%)$ patients. Esophageal tissues around the fistula appear to epithelialize in response to CRT, but the mechanisms behind this effect are unclear. The major pathology of esophagus cancer is squamous cell carcinoma, a tumor with relatively high radiosensitivity. We speculate that radiosensitivity might be critical in this phenomenon. In tissue repair, transforming growth factor- $\beta$ (TGF- $\beta$ ) is a key cytokine that stimulates the production of surrounding extracellular matrix and heals the injured tissue [12]. Given that radiation often induces TGF- $\beta$ signaling [13], we also speculate that this type of wound healing mechanism might underlie the epithelialization.

TEF formation in lung carcinoma is rarely reported. To our knowledge, this is the first report of primary lung cancer with TEF successfully treated with CRT. The therapeutic agents for TEF should be selected by carefully considering the patient's particular condition and the risk of aggravating the TEF. Though the usefulness and safety must be confirmed through further clinical research, CRT may prove to be effective as a palliative treatment for malignant TEF in lung cancer. 
Table 1. Laboratory tests on admission

\begin{tabular}{lll}
\hline & $\begin{array}{l}\text { On ad- } \\
\text { mission }\end{array}$ & $\begin{array}{l}\text { Reference } \\
\text { range }\end{array}$ \\
\hline White blood cells, /mm & & \\
Differential count, \% & 5,700 & $3,900-8,800$ \\
$\quad$ Neutrophils & & \\
$\quad$ Lymphocytes & 76 & $28-70$ \\
$\quad$ Monocytes & 21 & $20-58$ \\
$\quad$ Eosinophils & 1.0 & $0-12$ \\
Red blood cells, $\times 10^{4} / \mathrm{mm}^{3}$ & 2.0 & $0-5$ \\
Hemoglobin, g/dl & 399 & $418-564$ \\
Hematocrit, \% & 12.6 & $13.4-17.5$ \\
Platelet count, $\times 10^{4} / \mathrm{mm}^{3}$ & 37.7 & $39.5-52.1$ \\
Total protein, g/dl & 21.8 & $13.9-37.3$ \\
Albumin, g/dl & 7.2 & $6.8-8.3$ \\
Urea nitrogen, mg/dl & 3.0 & $3.8-5.2$ \\
Creatinine, mg/dl & 11.2 & $8.0-22.0$ \\
Sodium, mmol/l & 0.65 & $0.30-1.20$ \\
Potassium, mmol/l & 146.5 & $134.0-150.0$ \\
Chloride, mmol & 3.8 & $3.5-5.0$ \\
Calcium, mg/dl & 107.4 & $98.0-108.0$ \\
Lactate dehydrogenase, IU/l & 9.2 & $8.7-11.0$ \\
Alanine aminotransferase, IU/l & 602 & $107-220$ \\
Aspartate aminotransferase, IU/l & 37 & $10-34$ \\
Bilirubin total, mg/dl & 41 & $6-34$ \\
Glucose, mg/dl & 0.49 & $0.22-1.20$ \\
C-reactive protein, mg/dl & 81 & $60-110$ \\
Carcinoembryonic antigen, ng/ml & 13.35 & $0.00-0.30$ \\
Carbohydrate antigen 19-9, ng/ml & 1.8 & $0.0-5.2$ \\
Squamous cell carcinoma antigen, ng/ml & 6.9 & $0.0-36.8$ \\
Cytokeratin 19 fragment, ng/ml & 1.1 & $\leq 1.5$ \\
Progastrin-releasing peptide, pg/ml & 7.7 & $\leq 3.5$ \\
Neuron-specific enolase, ng/ml & 32.7 & $\leq 46.0$ \\
& 34 & $\leq 10$ \\
\hline & & \\
& & \\
& &
\end{tabular}



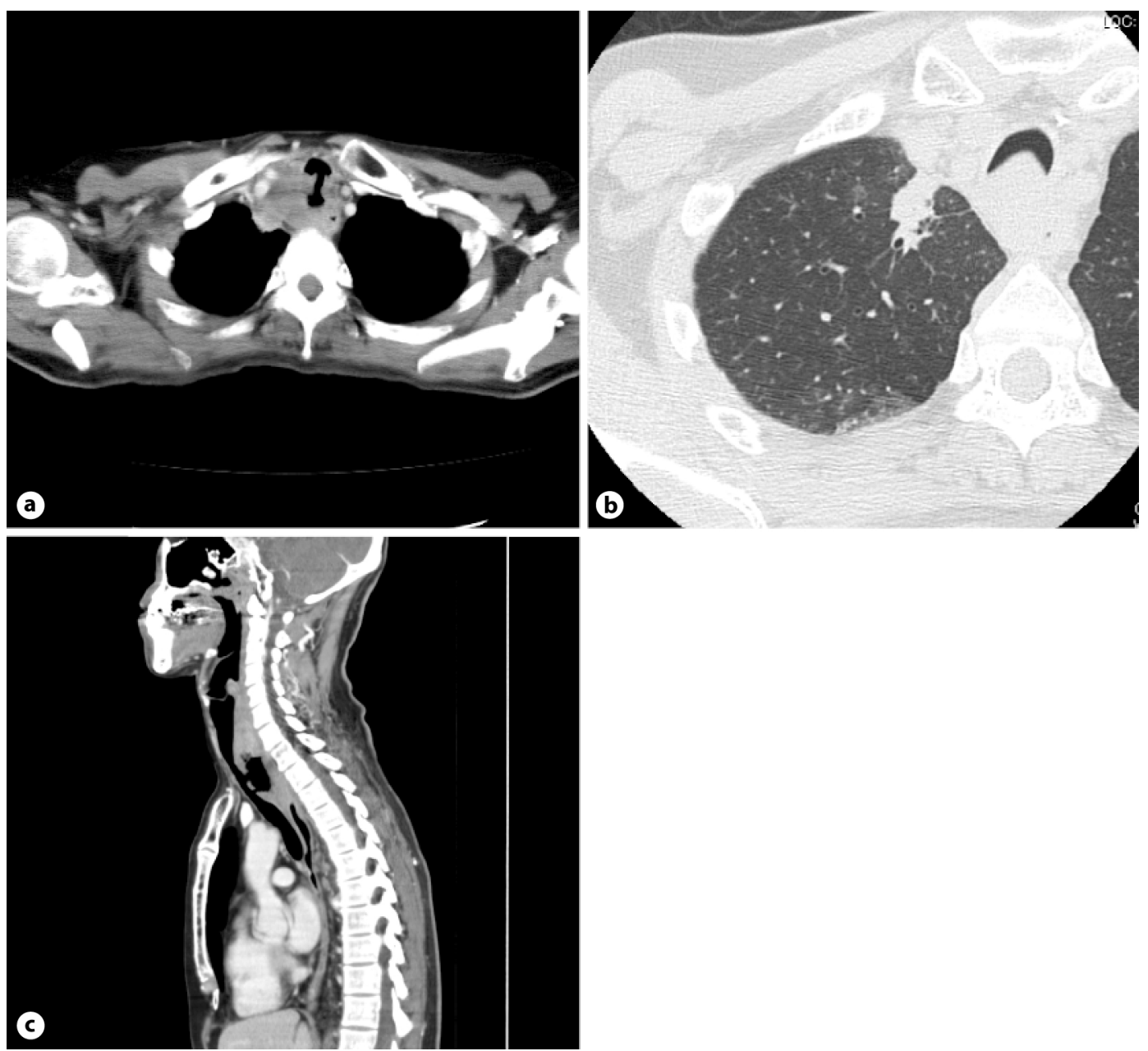

Fig. 1. An enhanced chest CT scan shows a small mass in the superior sulcus with invasion into the mediastinum $(\mathbf{a}, \mathbf{b})$. The sagittal view reveals a cavity in the mass and compression of the stenotic trachea by the mass $(\mathbf{c})$. 

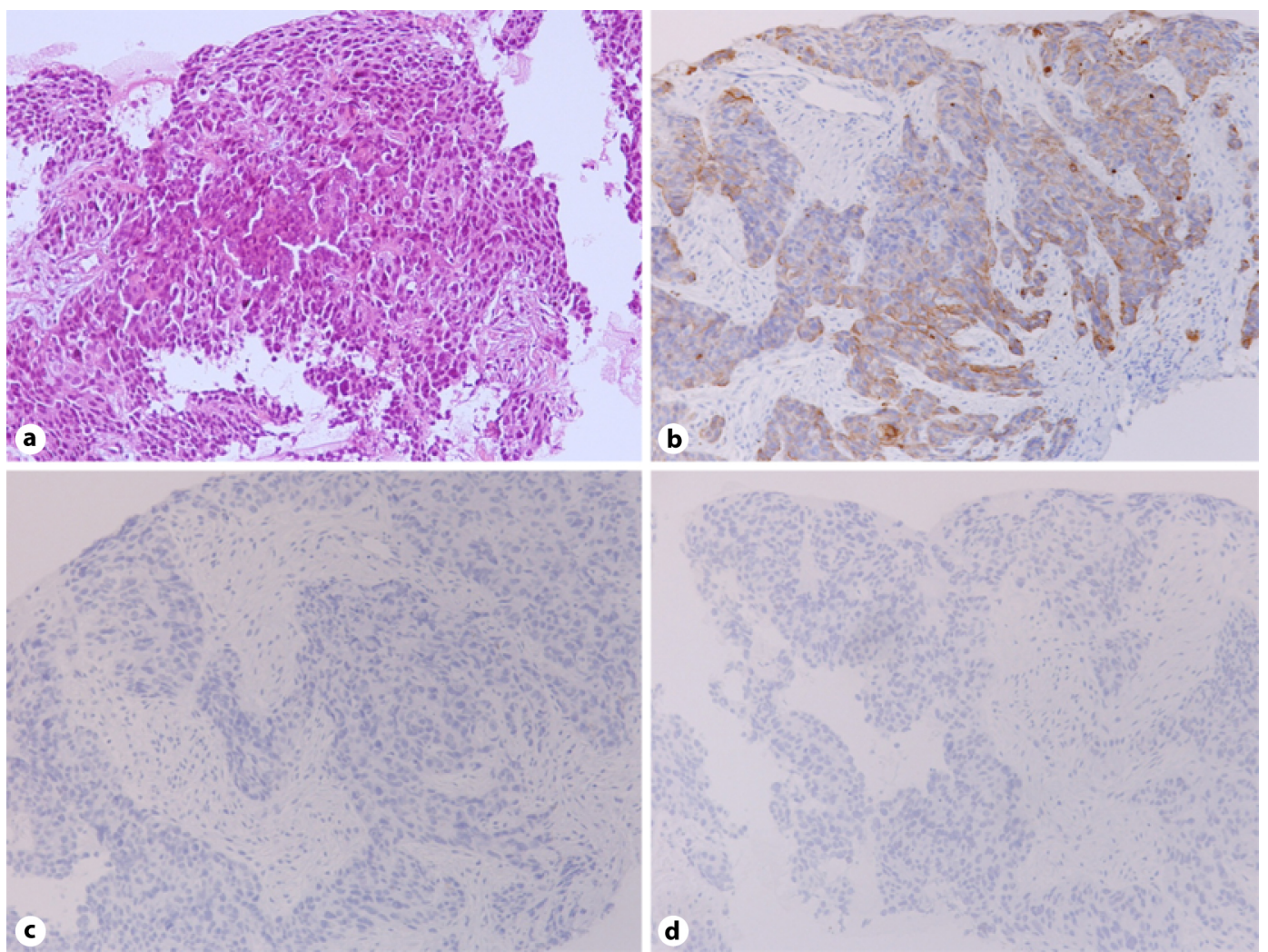

Fig. 2. Percutaneous needle biopsy specimen shows tumor cells with enlarged nuclei and scanty cytoplasm $(\mathrm{HE}, \times 100)$. Immunohistochemically, the tumor cells are positive for CK-7 (b) and negative for CK-14 (c) and TTF-1 (d) $(\times 400)$. 

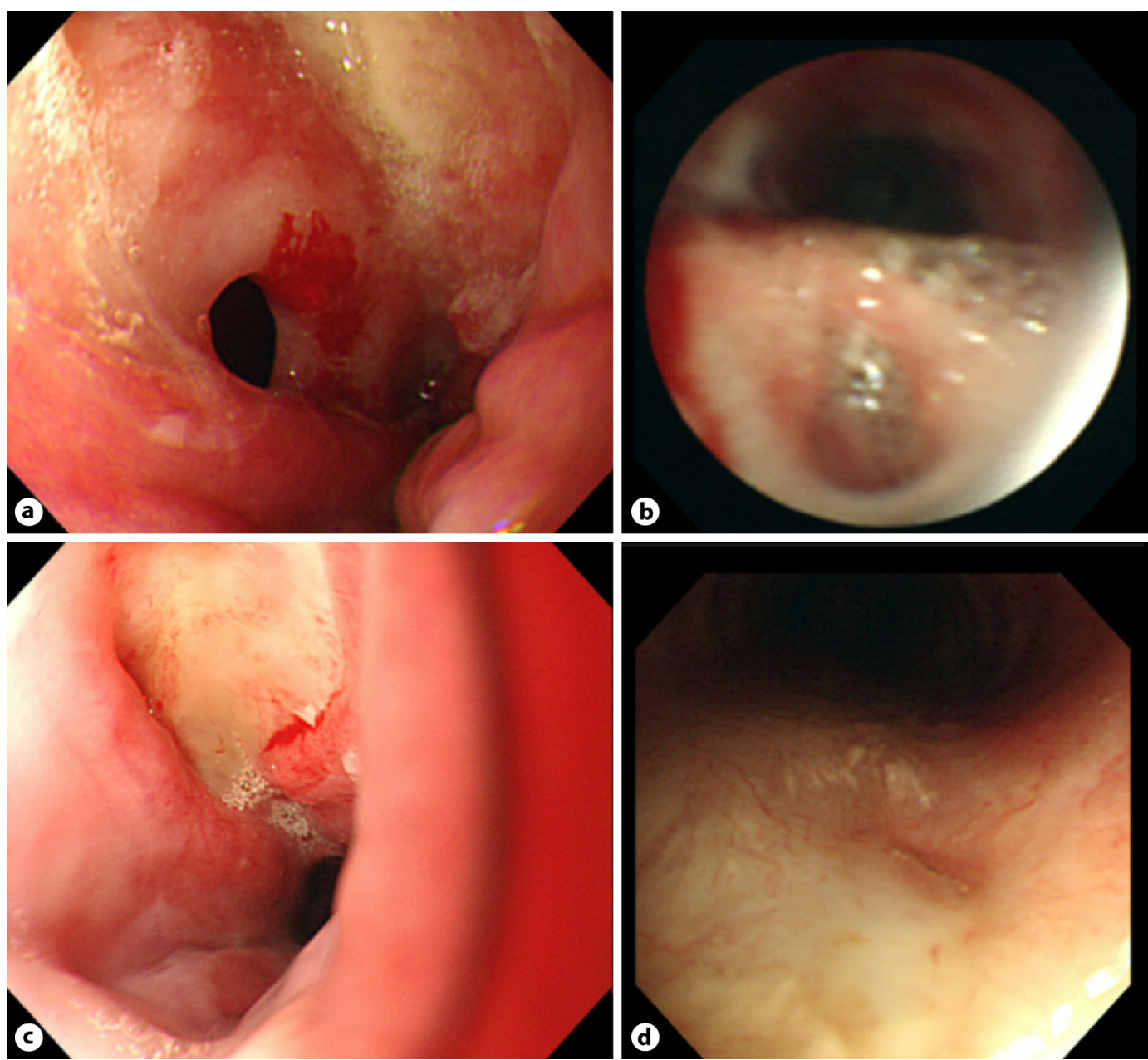

Fig. 3. Upper gastrointestinal endoscopy reveals the fistula located in the upper esophagus (a). Bronchoscopy shows a tracheal cavity communicating with the esophagus (b). A follow-up upper gastrointestinal endoscopy reveals a covering of epithelial tissue over the fistula (c), and bronchoscopy shows only one slit on the trachea $(\mathbf{d})$.

\section{References}

1 Fitzgerald RH, Bartles DM, Parker EF: Tracheoesophageal fistulas secondary to carcinoma of esophagus. J Thorac Cardiovasc Surg 1981;82:194-197.

2 Paul AK, Paul AS, Udaya BSP: Palliative care in lung cancer: ACCP evidence-based clinical practice guidelines (2nd edition). Chest 2007;132:3685-4035.

3 Ohtsu A, Yoshida S, Boku N, Fujii T, Oda Y, Miyata Y, Koba I, Muro K, Ohnaka O, Shimizu W, Ogino T, Abe $\mathrm{K}$ : Small cell of the esophagus with an esophago-mediastinal fistula successfully treated by chemoradiation therapy and intubation. Jpn J Clin Oncol 1993;23:372-377.

4 Malik SM, Krasnow SH, Wadleigh RG: Closure of fistulas with primary chemotherapy in patients with esophageal cancer. Cancer 1994;73:1321-1323.

5 Michael FR, Douglas JM: Tracheoesophageal fistula. Chest Surg Clin N Am 2003;13:271-289.

6 6 Lutz F, Edith T, Heinz S, Terrence JD, Georgios S: Management of malignant esophagotracheal fistulas with airway stenting and double stenting. Chest 1996;110:1155-1160. 
7 Tracey LE: Novel, targeted agents with thoracic radiation in the treatment of locally advanced non-small cell lung cancer. ASCO educational book, 2009.

$>8$ Herbert H, Louis F, William N, Thomas C, John H, William H, Jordan B, Ari B, Susan G, Eric H, Napoleone F, Gwen F, Beth R, Robert R, Fairooz K: Bevacizumab plus irinotecan, fluorouracil, and leucovorin for metastatic colorectal cancer. N Engl J Med 2004;350:2335-2342.

-9 Alan S, Robert G, Micheal CP, Julie B, Joan HS, Afshin D, Rogerio L, David HJ: Paclitaxel-carboplatin alone or with bevacizumab for non-small-cell lung cancer. N Engl J Med 2006;355:2542-2550.

10 Spigel DR, Hainsworth JD, Yardley DA, Raefsky E, Patton J, Peacock N, Farley C, Burris HA 3rd, Greco FA: Tracheoesophageal fistula formation in patients with lung cancer treated with chemoradiation and bevacizumab. J Clin Oncol 2010;28:43-48.

11 Manabu M, Atsushi O, Shin-ichi M, Kei M, Narikazu B, Satoshi I, Mitsuo S, Takashi O, Hisao T, Shigeaki Y: Concurrent chemoradiotherapy for esophageal carcinoma patients with malignant fistulae. Cancer 1999;86:1406-1412.

12 Wayne AB, Nancy AN: Transforming Growth factor $\beta$ in tissue fibrosis. N Engl J Med 1994;331:1286-1292.

13 Horatiu CD, Mohammad MS, Mansoor MA: Role of radiation-induced TGF- $\beta$ signaling in cancer therapy. Mol Cell Pharmacol 2009;1:44-56. 